\title{
Knowing that strawberries are red and seeing red strawberries: the interaction between surface colour and colour knowledge information
}

\author{
Inês Bramão ${ }^{\mathrm{a}, \mathrm{b}}$, Alexandra Reis ${ }^{\mathrm{a}}$, Karl Magnus Petersson ${ }^{\mathrm{a}, \mathrm{c}, \mathrm{d}}$ and Luís Faísca ${ }^{\mathrm{a}}$ \\ ${ }^{a}$ Cognitive Neuroscience Research Group, Centre for Biomedical Research (CBMR), University of Algarve, Faro, Portugal; ${ }^{b}$ Department \\ of Psychology, Lund University, Lund, Sweden; ${ }^{C}$ Max Planck Institute for Psycholinguistics, Nijmegen, The Netherlands; ${ }^{\mathrm{d}}$ Donders \\ Institute for Brain, Cognition and Behaviour, Radboud University, Nijmegen, The Netherlands
}

\begin{abstract}
This study investigates the interaction between surface and colour knowledge information during object recognition. In two different experiments, participants were instructed to decide whether two presented stimuli belonged to the same object identity. On the non-matching trials, we manipulated the shape and colour knowledge information activated by the two stimuli by creating four different stimulus pairs: (1) similar in shape and colour (e.g. TOMATO-APPLE); (2) similar in shape and dissimilar in colour (e.g. TOMATO-COCONUT); (3) dissimilar in shape and similar in colour (e.g. TOMATO-CHILI PEPPER) and (4) dissimilar in both shape and colour (e.g. TOMATOPEANUT). The object pictures were presented in typical and atypical colours and also in black-and-white. The interaction between surface and colour knowledge showed to be contingent upon shape information: while colour knowledge is more important for recognising structurally similar shaped objects, surface colour is more prominent for recognising structurally dissimilar shaped objects.
\end{abstract}

ARTICLE HISTORY

Received 16 June 2015

Accepted 19 April 2016

\section{KEYWORDS}

Object recognition; surface colour information; colour knowledge information; shape information

\section{Introduction}

Perceiving the colour red in a tomato and knowing that a tomato is red represent distinct cognitive operations. The surface colour of an object can be defined as the percept generated by the colour displayed in the object image (e.g. the colour red in a picture of a tomato), while colour knowledge refers to the semantic knowledge about the prototypical object colour (e. g. the knowledge that tomatoes are typically red). According to the "Shape + Surface" model, introduced by Tanaka and collaborators (Tanaka, Weiskopf, \& Williams, 2001), object recognition is jointly determined and facilitated by the bottom-up influence of perceptual surface colour and the top-down influence of colour knowledge. However, the way these two sources of colour information interact during object recognition is still unknown.

In a previous study, we showed that colour knowledge retrieval was dependent on appropriate surface colour information, suggesting that the bottom-up surface colour is more important during recognition than the top-down colour knowledge information (Bramão, Faísca, Petersson, \& Reis, 2010; see also, Huettig \& Altmann, 2011). In a word-picture verification task we manipulated the relationship between the colour and shape information provided by the word and by the picture. Subjects took longer to say that the name orange did not match the picture of a carrot, only if the carrot was presented in a typical colour format, suggesting that surface colour information was an important cue to trigger colour knowledge information during object recognition processes (Bramão et al., 2010). However, in a series of verification tasks, Joseph and collaborators (Joseph, 1997; Joseph \& Proffitt, 1996) manipulated the perceptual colour input independently of the colour knowledge and concluded that the top-down effect of colour knowledge is more important during object recognition than the bottom-up effect of surface colour. The authors found that a purple apple was more likely to be mistaken for a cherry than for a blueberry. The interference effect occurred because both apples and cherries are typically red, not because the apple was coloured in purple, the 
typical colour of a blueberry. The same result was obtained when uncoloured pictures were used, suggesting that the conceptual processing of colour does not depend on the presence of surface colour.

A potential confound in these studies is the role of shape information. Joseph and collaborators (Joseph, 1997; Joseph \& Proffitt, 1996) only used pairs of objects similar in shape (e.g. cherry and blueberry), in contrast, Bramão et al. (2010) used objects of dissimilar shape (e.g. carrot and orange). Because object recognition is essentially a shape-driven system (Biederman, 1987; Marr \& Nishihara, 1978), it is possible that the interaction between colour knowledge and surface colour information is contingent upon shape information. In addition, previous studies have suggested that colour information plays a prominent role during the recognition of non-diagnostic shaped objects, that is, similarly shaped objects, by acting as a cue to reduce the competition at the level of the object's structural representation (Laws \& Hunter, 2006; Price \& Humphreys, 1989; Wurm, Legge, Isenberg, \& Luebker, 1993). For example, Wurm et al. (1993) showed that prototypical images exhibit a smaller colour advantage than non-prototypical ones. This suggests that shape plays an important role mediating the interaction between the surface and the colour knowledge systems.

When we view an object, colour and shape information are processed in a parallel fashion (Gegenfurtner, 2003). At some point, this information must be combined to achieve a unitary representation of the visual world. One possibility is that this information is combined during the stage of structural description selection, where colour knowledge might act as a constraint to limit the range of candidate structural descriptions. According to the model proposed by Humphreys and colleagues (Humphreys, Riddoch, \& Quinlan, 1988), structural descriptions are long-term memories that specify the visual features of members belonging to familiar categories. It is still unknown whether these representations code for object shape only (e.g. tomatoes are round) or also for their associated colours (e.g. tomatoes are red). According to some researchers, the available experimental evidence showing that colour improves recognition should be interpreted as favouring the claim that colour knowledge is an integral part of the long-term structural description of colour diagnostic objects (Bramão et al., 2012; Tanaka et al., 2001). However, this argument appears to be in conflict with the neuropsychological evidence showing dissociations between the colour and shape knowledge systems (Luzzatti \& Davidoff, 1994; Miceli et al., 2001; Riddoch \& Humphreys, 1987). The question of whether colour knowledge is stored together with shape information at the level of structural description might have implications for the way surface colour information and colour knowledge interact during object recognition. If shape and colour knowledge are stored together, then when the visual system is presented with an object, colour knowledge is automatically accessed together with shape information. In such a system, the activation of colour knowledge is more likely to be independent of the surface colour displayed in the object picture. That is, even if the objects are coloured in an incongruent surface colour, colour knowledge is automatically accessed together with shape. If this is the case, the top-down effect of colour knowledge might be stronger than the bottom-up effect of surface colour. On the other hand, if we consider a system where shape and colour are stored independently, it is possible to access shape information without colour knowledge retrieval. In this circumstance, it is more likely that surface colour can act as a cue for colour knowledge retrieval in a bottom-up fashion.

In this paper we aim to clarify the interaction between the colour knowledge and surface colour systems. Two experiments were carried out. In the first experiment we used a word-picture (WP) verification task and in the second experiment we used a picture-word (PW) verification task. Previous studies have shown differences in retrieving colour knowledge from verbal (words) and visual (pictures) modalities. The current idea in the literature is that the retrieval of colour knowledge is more effective when prompted by object names compared to pictures (Huettig \& Altmann, 2011; Lloyd-Jones \& Nakabayashi, 2014; Naor-Raz, Tarr, \& Kersten, 2003; however see Nijboer, van Zandvoort, \& de Haan, 2006). Furthermore, word and picture naming may involve different cognitive operations (Humphreys, Lloyd-Jones, \& Fias, 1995; Vitkovitch, Cooper-Pye, \& Leadbetter, 2006). For example, Humphreys et al. (1995) suggested that picture primes compared to word primes are more likely to activate competing representations. Thus, PW verification might enhance the colour knowledge effects because the primes (pictures) are more likely to activate competing representations and also because the targets 
(words) are more likely to activate colour knowledge information.

The two verification tasks of the current study contain two different types of trials: matching, where the two presented stimuli belong to the same object identity, and non-matching trials, where the two presented stimuli did not match. On the non-matching trials, the first stimulus (distractor) is expected to activate shape and colour knowledge information that might interfere with the shape and colour knowledge information provided by the second stimulus (target). When the processing system is confronted with a visual input, several long-term structural descriptions, belonging to objects that potential match the visual input, are activated. Thus, the distractor is expected to activate long-term structural descriptions of objects that share shape and colour knowledge. If, on the nonmatching trials, the colour and shape information activated by the distractor match the colour and shape information activated by the target, more interference is expected and, consequently longer response times (Bramão et al., 2010; Joseph, 1997; Joseph \& Proffitt, 1996). In order to test whether colour knowledge is automatically activated with shape information, we independently manipulated the shape and the colour knowledge information activated by the two stimuli. The target stimulus could be preceded by (1) a distractor similar in shape and in prototypical colour (the shape-colour distractor - e.g. the word/picture TOMATO followed by a picture/word of an APPLE); (2) a distractor similar in shape, but dissimilar in prototypical colour (the shape distractor - e.g. the word/picture TOMATO followed by a picture/word of an COCONUT); (3) a distractor dissimilar in shape, but similar in prototypical colour (the colour distractor - e.g. the word/picture TOMATO followed by a picture/word of a CHILI PEPPER) and (4) a distractor dissimilar in shape and in prototypical colour (the control distractor - e.g. the word/picture TOMATO followed by a picture/word of a PEANUT). To evaluate the interaction between surface colour information and colour knowledge information, the surface colour similarity between the distractor and the target were independently manipulated. To this end, the object pictures were presented in typical and atypical colours and also in black-andwhite. The surface colour between the distractor and the target were similar if the perceptual colour of the picture was congruent with the typical colour of the word (e.g. the word TOMATO (distractor) followed by a picture of a RED COCUNUT (target) in the WP verification task or the picture of a RED COCUNUT (distractor) followed by the word TOMATO (target) in the PW verification task). If colour and shape are stored together in the object's structural description, then colour knowledge is expected to be automatically activated together with shape information and result in a colour knowledge interference effect (longer response times on the non-matching trials whenever the colour knowledge information between the two stimuli are similar), independently of the shape information activated by the two stimuli. However, if there are separate representations for shape and colour knowledge, we expect to observe colour knowledge interference only when the shape of the distractor and the target are similar. In these circumstances, colour knowledge is expected to be activated in order to resolve the increased competition due to similar shape information. Moreover, and because the top-down effects of colour knowledge information might have a prominent role resolving this high competition, we expect to observe minor interference from the surface colour information. Conversely, when shape information is dissimilar, we do not expect colour knowledge to be automatically recruited, because participants can base their responses upon shape information. In this situation, the retrieval of colour knowledge might be triggered by surface colour in a bottom-up fashion. Thus, we expect to observe a bottom-up surface colour interference effect, that is, subjects will take longer deciding that the two stimuli do not match when the prototypical colour of the distractor is congruent with the surface colour of the target stimulus.

Finally, we tested both line-drawings and photographs. In our previous study we used photographs (Bramão et al., 2010), while Joseph et al. (1996, 1997) used line-drawings. There is evidence that object recognition is faster for photographs than line-drawings (Brodie, Wallace, \& Sharrat, 1991; Price \& Humphreys, 1989) and Uttl and colleagues (Uttl, Graf, \& Santacruz, 2006) argue that line-drawings are typically viewed as representations of object classes, types, whereas photographs are viewed as individual objects, tokens. Moreover, the colour of a line-drawing is a non-natural colour, while the colour of a photograph picture is more naturalistic and harder to verbalise. A black-and-white line-drawing of a pig can be more easily recognised by human subjects if it is coloured in pink, even if 
the hue of the colour used is very different from the natural one. To study at which level of the object naming processes colour and photographic details exert an effect during recognition, Zannino and colleagues (Zannino et al., 2010) contrasted the performance of a group of Alzheimer patients with an age-matched control group in a naming task of line-drawings and photographs presented in colours and in black-and-white. The authors suggested that the effect of non-naturalistic colours in line-drawings arise at the semantic level, while the effect of adding natural colours to photographs arise at an early visual processing stage. The presence of easily nameable non-naturalistic colours might have acted as a verbal-semantic clue. Moreover, this result was more pronounced in the Alzheimer patients, which are known to suffer from major semantic impairment (Hodges, Salmon, \& Butters, 1992). Thus, we expect to find greater colour knowledge interference effects when objects are presented as line-drawings.

\section{Experiment 1 (word-picture verification)}

\subsection{Material and methods}

\subsubsection{Subjects}

Thirty right-handed subjects volunteered to participate in the experiment (mean age $[ \pm S D]=22.2 \pm$ 5.6 years, range 17-43 years; mean years of education $[ \pm S D]=14.2 \pm 1.1$ years, range $13-18$ years; 9 males and 21 females). All subjects were Portuguese native speakers with normal or corrected-tonormal vision; none report a history of colour vision problems, head injury, or other neurological or psychiatric problems.

\subsubsection{Stimuli}

Forty-eight objects, belonging to the semantic category of fruit and vegetables, were selected. Two versions of the 48 objects were created: a blackand-white line-drawing and a black-and-white photograph. The line-drawing version of each object was selected from the picture database at the Max Planck Institute for Psycholinguistics and some were selected via an Internet image search using the Google search engine. All the line-drawings were normalised using Adobe Photoshop CS2 to have the same qualities (orientation, size, line thickness, etc.). The stimuli were coloured in their typical colour. Each line-drawing was matched with a photograph as similar as possible in terms of colour, shape, size and orientation. The photographs were selected from the Focus Multimedia CD Photo Library, some were selected from the set of Reis and colleagues (Reis, Faísca, Ingvar, \& Petersson, 2006), and some were selected via an Internet image search using the Google search engine. The colour of each photograph was arranged, using Adobe Photoshop CS2, to be as similar as possible with the correspondent line-drawing. A black-andwhite version of each stimulus was created using the greyscale mode tool of Adobe Photoshop CS2, which preserves the luminance while discarding the colour.

The 48 stimuli were grouped into 12 sets, such that each target stimulus (e.g. TOMATO) had (1) a shape-colour distractor (e.g. APPLE - similar both in shape and colour); (2) a shape distractor (e.g. COCONUT - similar in shape and dissimilar in colour) and (3) a colour distractor (e.g. CHILI PEPPER - similar colour and dissimilar in shape). Additionally, a control distractor stimulus (e.g. PEANUT - dissimilar in both shape and colour information) was also included in each set. The control distractor stimulus was a distractor object from one of the other sets and was selected so that its prototypical colour would be the same as the prototypical colour of the shape distractor. Each set was created according to our intuition and afterwards two preliminary experiments objectively established (1) the prototypical colour of each object and (2) the shape and prototypical colour similarity between the object pairs. The complete procedure of these preliminary experiments can be consulted in the supplemental online material. Accordingly, a shape distractor should be an object that was similar in shape to the target, but dissimilar in prototypical colour. In the same way, a colour distractor is similar in prototypical colour to the target object, but dissimilar in shape. Finally, a shape-colour distractor is similar both in shape and prototypical colour to the target object. Thus, it was necessary to objectively establish the shape and colour knowledge similarity or dissimilarity between the target and the shape and colour distractor. Likewise, we needed to establish shape and prototypical colour similarity between the shape-colour distractor and the target. Finally, it was necessary to establish (1) that the shape similarity between target and shape distractor were equivalent to the shape similarity between target and shape-colour distractor and (2) that the colour knowledge similarity between 
target and colour distractor was equivalent to the colour knowledge similarity between target and shape-colour distractor. This procedure ensured that the interference observed between distractor and target was caused by colour and/or shape similarity. The complete stimuli material with the 12 sets can be consulted in Table 1 and some exemplars of the stimuli can be seen in Figure 1.

To create the incongruent colour version of the shape and control distractors, we selected the surface colour of the target and paste onto the surface of these stimuli, using the replacement colour tool in Adobe Photoshop CS2. In the same way, the incongruent colour version of the target, shape-colour and colour distractor was created by selecting the surface colour of the control distractor and pasting the colour onto these stimuli. This procedure ensured that congruent and incongruent coloured objects were matched for colour frequency and luminance. In total six different versions of each object were created.

\subsubsection{Procedure}

A computerised verification task was designed in which an object picture was preceded by an object name. Participants had to decide whether the name and the picture matched. The verification task comprised 864 trials. Because we were mainly interested in the non-matching trials, $2 / 3$ were non-matching trials (576 trials in which the name and the picture did not match) and $1 / 3$ were matching trials (288 trials in which the name and the picture matched). On the matching trials, each object version was presented only one time (48 objects $\times 6$ presentation versions). On the non-

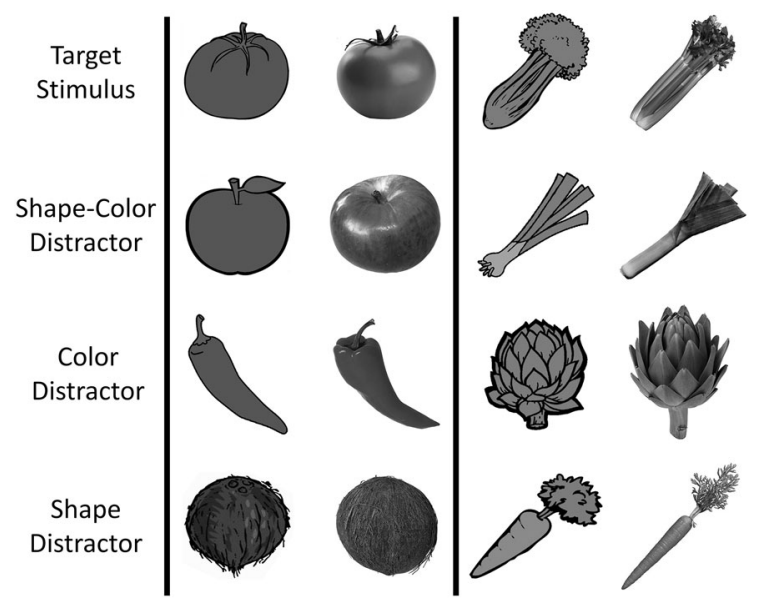

Figure 1. Example of the stimuli used in the study. matching trials, there were 144 trials of each type: target preceded by the shape-colour distractor; target preceded by the shape distractor, target preceded by the colour distractor, and target preceded by the control distractor ( 12 object sets $\times 6$ presentation versions $\times 4$ trial types). In order to increase the number of observations per condition and per participant, we also included trials where the shape-colour distractor served as target. In this way, each distractor version was presented twice, one time preceded by the word corresponding to the target (e.g. TOMATO) and another time preceded by the shape-colour distractor word serving as target (e.g. APPLE) (144 trials $\times 2$ target versions).

The Presentation 0.7 software (http://nbs.neurobs.com/presentation) was used to display the stimuli on a computer screen (size, $17^{\prime} /$; spatial resolution, $1024 \times 768$; colour resolution, 24 bits) and to register the response times. Each trial started with a fixation cross presented at the centre of the screen for $1250 \mathrm{~ms}$. After the fixation cross, the object name (font Arial, font size 70) was presented for $1000 \mathrm{~ms}$, followed by the presentation of the object picture $(633 \times 498$ pixels $)$ for $50 \mathrm{~ms}$. After the object picture presentation a visual mask was presented for $10 \mathrm{~ms}$. The trial ended with the participant's response (Figure 2). If participant's response did not occur during a time window of $1500 \mathrm{~ms}$ a new trial started. Participants were instructed to decide as accurately and as quickly as possibly whether the name and the picture matched by pressing one of the two response keys of the keyboard (half of the participants used the right/left hand for "yes"/"no" and the other half for "no"/ "yes"). The 864 trials were split into 4 blocks of 216 trials each. Both blocks and trials within blocks were randomised across participants. Participants were given a break of 5 minutes between blocks.

To ensure that all subjects appropriately match the word labels to the corresponding figures a training phase was included. Before subjects were engage in the verification task, each object version (congruent, incongruent and absent surface colour version of each line-drawing and photograph) was shown to the subjects with the corresponding word label on top of the screen. After that, participants were engaged in an object picture naming task for each object version. At the end of the naming task, feedback was given to the subjects (for a similar procedure see Joseph \& Proffitt, 1996). The complete experimental procedure took about 90 minutes. 
Table 1. The 12 sets of objects used in the study.

\begin{tabular}{|c|c|c|c|c|c|c|c|c|}
\hline \multirow[b]{2}{*}{$\begin{array}{l}\text { Target } \\
\text { object }\end{array}$} & \multirow[b]{2}{*}{$\begin{array}{c}\text { Shape-colour } \\
\text { distractor }\end{array}$} & \multirow[b]{2}{*}{$\begin{array}{c}\text { Colour } \\
\text { distractor }\end{array}$} & \multicolumn{2}{|c|}{ Surface colour } & \multirow[b]{2}{*}{$\begin{array}{c}\text { Shape } \\
\text { distractor }\end{array}$} & \multirow[b]{2}{*}{$\begin{array}{c}\text { Control } \\
\text { distractor }\end{array}$} & \multicolumn{2}{|c|}{ Surface colour } \\
\hline & & & Congruent & Incongruent & & & Congruent & Incongruent \\
\hline Blueberry & Passion Fruit & Mulberry & Purple & Orange & Pumpkin & Cashew & Orange & Purple \\
\hline Celery & Leek & Artichoke & Green & Orange & Carrot & Pumpkin & Orange & Green \\
\hline Cucumber & Asparagus & Broccoli & Green & Yellow & Banana & Melon & Yellow & Green \\
\hline Lemon & Melon & Corn & Yellow & Red & Radish & Watermelon & Red & Yellow \\
\hline Meddler & Apricot & Papaya & Orange & Red & Cherry & Raspberry & Red & Orange \\
\hline Olive & Lime & Peas & Green & Orange & Mango & Carrot & Orange & Green \\
\hline Orange & Peach & Cashew & Orange & Red & Pomegranate & Chili pepper & Red & Orange \\
\hline Pepper & Pear & Lettuce & Green & Purple & Eggplant & Beet & Purple & Green \\
\hline Plum & Beet & Grapes & Purple & Brown & Onion & Potato & Brown & Purple \\
\hline Strawberry & Raspberry & Watermelon & Red & Brown & Acorn & Coconut & Brown & Red \\
\hline Tomato & Apple & Chili pepper & Red & Brown & Coconut & Peanut & Brown & Red \\
\hline Walnut & Potato & Peanut & Brown & Green & Kiwi & Peas & Green & Brown \\
\hline
\end{tabular}

Notes: Each set was composed by a target object, a shape-colour distractor (an object similar in shape and similar in colour), a shape distractor (an object similar in shape and dissimilar in colour), a colour distractor (an object dissimilar in shape and similar in colour) and a control distractor (an object both dissimilar in colour and in shape). Each object was presented in a line-drawing and in a photograph version in three different modalities: congruent and incongruent colour, as well as in a black-and-white.

\subsubsection{Data analysis}

The verification time was quantified as the time between the onset of the target and the participant's response. The verification time of the nonmatching trials were analysed by subject $(F 1)$ and by stimulus (F2). We only report the results from the non-matching trials. The results of the matching trials can be consulted in the supplemental online material. Overall, participants were able to correctly verify almost all stimuli, and we focused our analysis on the verification times from the correct trials with latencies within 2.5 standard deviations of the mean for each participant and condition. We excluded from the analysis verification times corresponding to trials where participants responded more than once, incorrect responses, as well as long and short verification times.
A minimum $F(\min F)$ was calculated from the $F 1$ and $F 2$ analyses. We report $F 1, F 2$ and $\min F$ values; however, our conclusions are based on the conservative $\min F$ analysis. This approach was taken to ensure the generalizability of results over both subject and stimulus domains (see also Clark, 1973; Raaijmakers, 2003; Raaijmakers, Schrijnemakers, \& Gremmen, 1999). The non-significant main effects and interactions are not reported. The verification times of the non-matching trials were analysed with a repeated-measures ANOVA including the within-subject/stimulus factors: Shape Similarity (similar vs. dissimilar shape), Colour Knowledge Similarity (similar vs. dissimilar colour knowledge), Surface Colour Information (congruent vs. incongruent vs. absent surface colour), and Stimulus Type (line-drawings vs. photographs).

(a) Experiment 1 - Word-Picture Verification
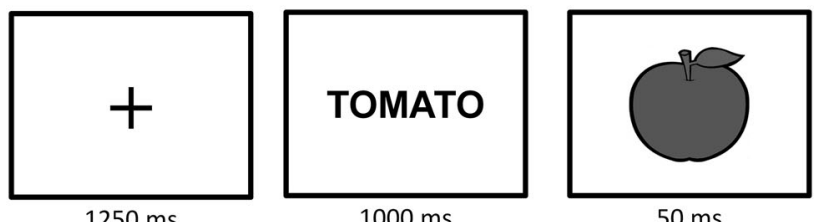

$50 \mathrm{~ms}$

Experiment 2 - Picture-Word Verification

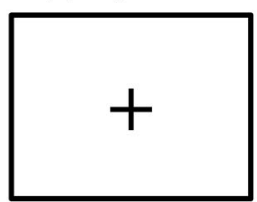

$1250 \mathrm{~ms}$

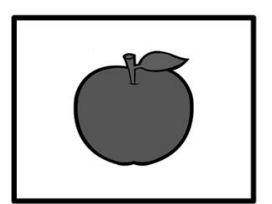

$50 \mathrm{~ms}$

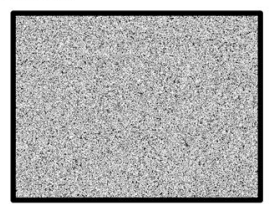

$10 \mathrm{~ms}$

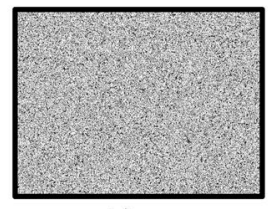

$10 \mathrm{~ms}$

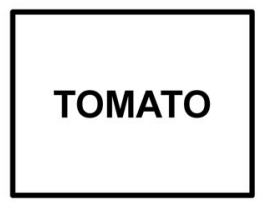

RESPONSE

Figure 2. Structure of a non-matching trial for Experiments $1(A)$ and 2(B). Each trial started with the presentation of a fixation point, followed by the distractor and the target. The trial ended with participant's response. A - Experiment 1 (WP verification task): the distractors were presented as word names and the targets as pictures. B - Experiment 2 (PW verification task): the distractors were presented as pictures and the targets as word names. 
Table 2. Significant main effects and interactions found in Experiment 1 (word-picture verification).

\begin{tabular}{|c|c|c|c|}
\hline & $\begin{array}{l}\text { Subject } \\
\text { (F1) }\end{array}$ & $\begin{array}{l}\text { Stimulus } \\
\text { (F2) }\end{array}$ & $\begin{array}{l}\text { Minimum } \\
F(\min F) \\
\end{array}$ \\
\hline Shape & $<0.001$ & $<0.001$ & $<0.001$ \\
\hline Colour knowledge & $<0.001$ & $<0.001$ & 0.001 \\
\hline Surface colour & $<0.001$ & $<0.001$ & 0.002 \\
\hline Stimulus type & $<0.001$ & $<0.001$ & $<0.001$ \\
\hline Shape $\times$ colour knowledge & $<0.001$ & 0.001 & $<0.001$ \\
\hline $\begin{array}{l}\text { Shape } \times \text { colour knowledge } \times \\
\text { surface colour }\end{array}$ & $<0.001$ & 0.02 & 0.04 \\
\hline $\begin{array}{l}\text { Colour knowledge } \times \text { surface } \\
\text { colour } \times \text { stimulus type }\end{array}$ & $<0.001$ & 0.001 & 0.01 \\
\hline \multicolumn{4}{|l|}{ Follow-up comparisons } \\
\hline \multicolumn{4}{|l|}{ Similar shape condition } \\
\hline Colour knowledge & $<0.001$ & 0.001 & $<0.001$ \\
\hline Surface colour & $<0.001$ & $<0.001$ & 0.004 \\
\hline $\begin{array}{l}\text { Colour knowledge } \times \text { surface } \\
\text { colour }\end{array}$ & $<0.001$ & 0.002 & 0.03 \\
\hline $\begin{array}{l}\text { Colour knowledge } \times \text { surface } \\
\text { colour } \times \text { stimulus type }\end{array}$ & 0.002 & 0.001 & 0.06 \\
\hline \multicolumn{4}{|l|}{ Dissimilar shape condition } \\
\hline Surface colour & $<0.001$ & 0.002 & 0.006 \\
\hline Stimuli type & $<0.001$ & 0.008 & 0.01 \\
\hline $\begin{array}{l}\text { Colour knowledge } \times \text { surface } \\
\text { colour }\end{array}$ & 0.01 & 0.08 & 0.1 \\
\hline
\end{tabular}

\subsection{Results}

In total, $9.2 \%$ of the verification times were excluded from the analysis ( $0.8 \%$ two or more responses; $4.7 \%$ incorrect; $3.5 \%$ long; $0.2 \%$ short). Table 2 shows a summary of the significant main effects and interactions found in the analysis. The response time analysis showed a significant shape similarity effect $(F 1(1,29)=364.4, p<.001 ; F 2(1,11)=61.2, p<.001$; $\min F(1,15)=52.4, p<.001)$ - when shape information provided by the picture and the previous word were similar there were longer response times $[640 \pm 15$ (Mean RT \pm SD)] compared to the dissimilar case $(555 \pm 13)$. The main effect of colour knowledge similarity was also significant $(F 1(1,29)$ $=237.5, p<.001 ; F 2(1,11)=18.6, p<.001 ; \min F(1$, $13)=17.2, p=.001)-$ when colour knowledge activated by the word and the picture were similar there were longer response times $(620 \pm 14)$ than in the dissimilar case $(575 \pm 13)$. We also observed a significant surface colour information effect $(F 1$ ( 2 , $58)=24.8, p<.001 ; F 2(2,22)=17.2, p<.001 ; \min F$ $(2,53)=10.1, p=.002)-$ a post-hoc comparison (Tukey HSD) for the subject analysis showed longer response times when the surface colour information displayed in the image was congruent with the colour knowledge activated by the previous presented object name $(609 \pm 14)$ compared to both incongruent $(588 \pm 14 ; p<.001)$ or absent $(596 \pm$ $14 ; p<.001)$ surface colour information. Moreover, the main effect of stimulus type was also significant $(F 1(1,29)=28.5, p<.001 ; F 2(1,11)=36.3, p<.001$; $\min F(1,35)=16.0, \quad p<.001) \quad$ - the photographs $(603 \pm 14)$ lead to longer response times compared to the line-drawings $(592 \pm 13.6)$. The two-way interaction between shape and colour knowledge was significant $(F 1(1,29)=207.8, p<.001 ; F 2(1,11)=$ 22.2, $p=.001 ; \min F(1,13)=20.0, p<.001)$. A posthoc comparison (Tukey HSD) for the subject analysis showed that when shape information between the object word and the picture was dissimilar there was no colour knowledge interference (similar colour knowledge $=556 \pm 13$; dissimilar colour knowledge $=553 \pm 13 ; p=.87$ ). On the other hand, when shape information between the two stimuli was similar there was a colour knowledge interference effect (similar colour knowledge $=684 \pm 16.2$; dissimilar colour knowledge $=596 \pm 14 ; p<.001$ ). Finally, the three-way interactions between shape similarity, colour knowledge similarity and surface colour information $(F 1(2,58)=9.2, p<.001 ; F 2(2$, $22)=5.1, p=.02 ; \min F(2,48)=3.3, p=.04 ;$ Figure 3$)$ and between colour knowledge similarity, surface colour information and stimulus type $(F 1(2,58)=$ 7.9, $p<.001 ; F 2(2,22)=9.2, p=.001 ; \min F(2,62)=$ $4.8, p=.01$; Figure 4) were also significant. To further explore these three-way interactions, we performed two separate analyses for each shape similarity dimension. The results from these two analyses are reported below.

\subsubsection{Similar shape}

The results showed a significant colour knowledge similarity effect $(F 1(1,29)=271.7, p<.001 ; F 2(1$, $11)=21.9, p=.001 ; \min F(1,13)=20.3, p<.001)-$ when colour knowledge was similar we observed longer response times $(684 \pm 16)$ compared to the dissimilar case $(596 \pm 13)$. The main effect of surface colour information was also significant ( $F 1$ $(2,58)=9.2, p<.001 ; F 2(2,22)=15.6, p<.001 ; \min F$ $(2,76)=5.8, p=.004)-$ a post-hoc comparison (Tukey HSD) for the subject analysis showed that the response time was superior for trials with congruent surface colour $(651 \pm 14)$ than for trials with incongruent $(631 \pm 15 ; p<.001)$ or absent $(638 \pm$ $15 ; p=.02)$ surface colour information. The twoway interaction between the factors colour knowledge similarity and surface colour information was also significant $(F 1(2,58)=8.6, p<.001 ; F 2(2,22)=$ 8.9, $p=.002 ; \min F(2,65)=3.5, p=.03)$. A post-hoc comparison (Tukey HSD) for the subject analysis showed that, when the colour knowledge between the stimuli was similar, there was no surface colour interference effect (congruent surface colour = 


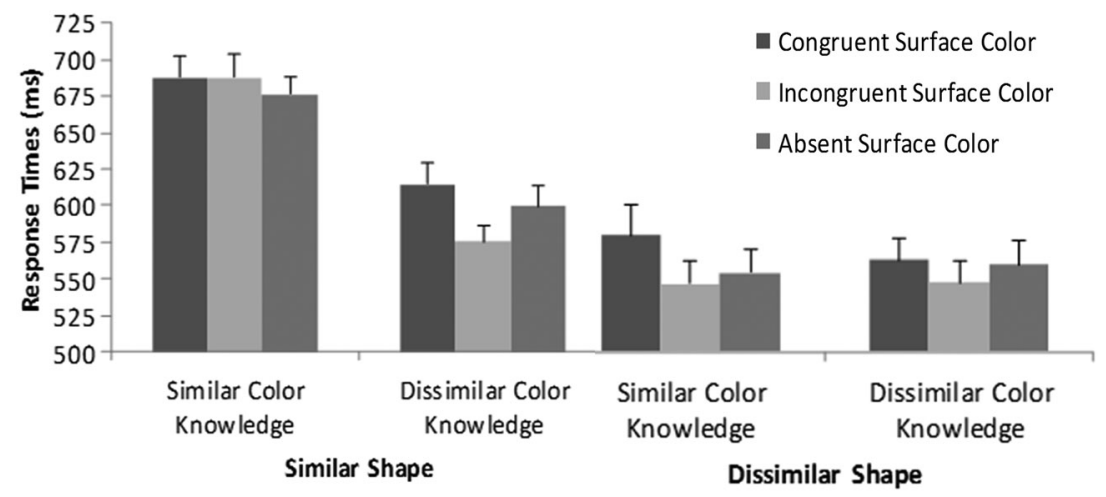

Figure 3. Three-way interaction between shape similarity, colour knowledge similarity and surface colour $(F 1(2,58)=9.2$, $p<.001 ; F 2(2,112)=4.9, p=.009 ; \min F(2,170)=3.2, p=.04)$ found in Experiment 1 (WP verification task). Bars represent one standard error.

$687 \pm 15$; incongruent surface colour $=688 \pm 19$; absent surface colour $=676 \pm 17 ; p>.6)$. However, when the colour knowledge between the stimuli was dissimilar subjects a surface colour interference was observed - when the surface colour was incongruent there was less interference $(575 \pm 12)$ compared to congruent $(614 \pm 14.9 ; p<.001)$ or absent $(600 \pm 15 ; \quad p=.02)$ surface colour information. Additionally, the three-way interaction between colour knowledge similarity, surface colour information and stimulus type was also marginally significant $(F 1(2,58)=7.2, p=.002 ; F 2(2,22)=8.8, p=.001$; $\min F(2,10)=3.9, p=.06$; Figure 5$)$. In order to further explore this three-way interaction two separate analysis were conducted for each stimulus type. The two-way interaction between colour knowledge and surface colour was only found for the photos ( $F 1$ $(2,58)=14.4, p<.001 ; F 2(2,22)=15.2, p<.001 ; \min F$ $(2,65)=7.4, p=.002$ ). A post-hoc comparison (Tukey $\mathrm{HSD}$ ) showed that when the colour knowledge were similar there was no surface colour interference (congruent surface colour $=684 \pm 15$; incongruent surface colour $=705 \pm 20$; absent surface colour $=$ $680 \pm 16 ; p>.2)$. However, when the colour knowledge was dissimilar there was a surface colour interference effect - there was less interference when the surface colour was incongruent $(570 \pm 13)$ compared to congruent $(626 \pm 17 ; p<.001)$ or absent $(606 \pm 15$; $p=.01)$ surface colour information.

\subsubsection{Dissimilar shape}

The results showed a significant main effect of surface colour information $(F 1(2,58)=17.8$, $p<.001 ; F 2(2,22)=8.4, p=.002 ; \min F(2,44)=5.7$, $p=.006$ ) - a post-hoc comparison (Tukey HSD) for the subject analysis showed that the response times were longer for trials with congruent $(567 \pm$ 14) than for trials with incongruent $(544 \pm 13$; $p<.0014)$ or absent $(553 \pm 12.9 ; p=.001)$ surface colour information. There was also a main effect of

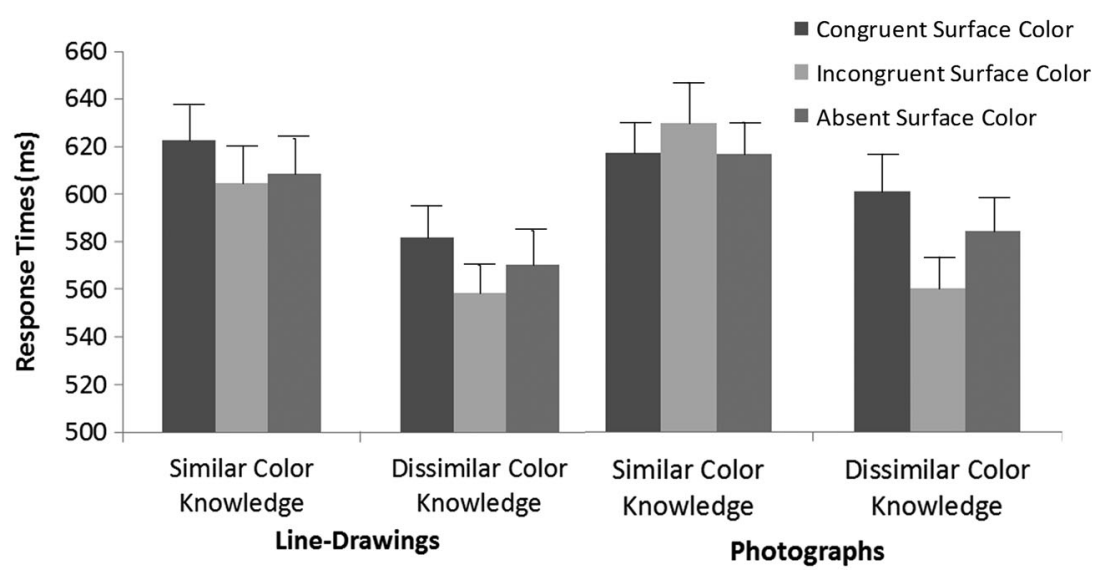

Figure 4. Three-way interaction between colour knowledge similarity, surface colour and stimulus type $(F 1(2,58)=7.9, p$ $<.001 ; F 2(2,112)=5.2, p=.007 ; \min F(2,170)=3.4, p=.04)$ found in Experiment 1 (WP verification task). Bars represent one standard error. 


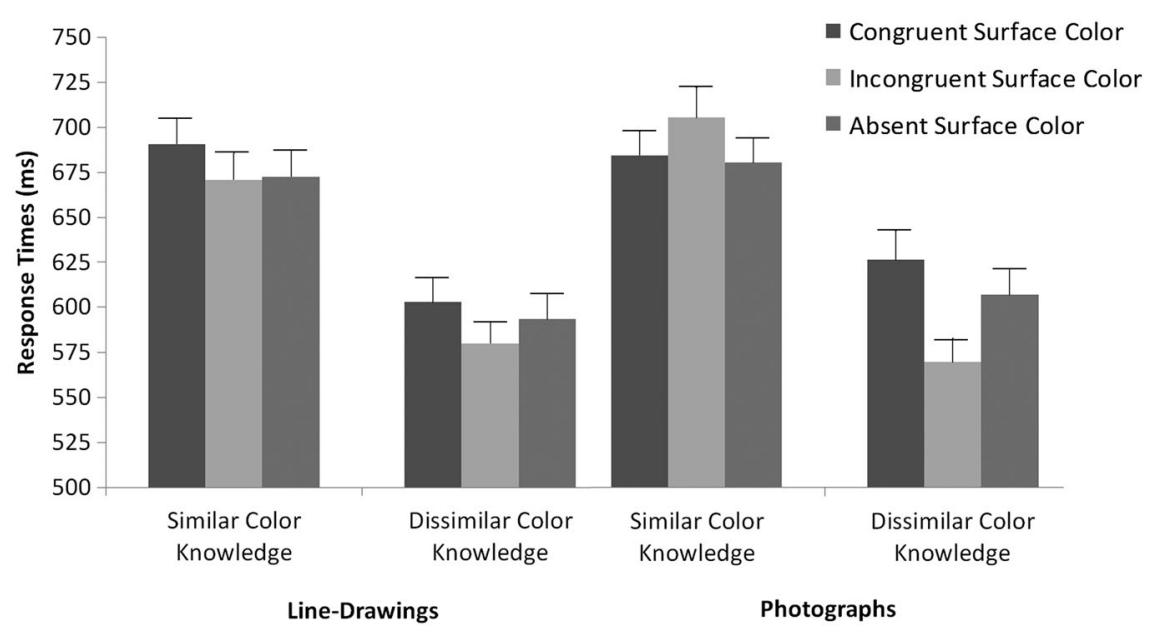

Figure 5. Three-way interaction between colour knowledge similarity, surface colour and stimuli type $(F 1(2,58)=7.2, p$ $=.002 ; F 2(2,68)=5.2, p=.007 ; \min F(2,125)=3.0, p=.05)$ found in Experiment 1 (WP verification task) for the similar shape information condition. Bars represent one standard error.

stimulus type $(F 1(1,29)=24.9, p<.001 ; F 2(1,11)=$ 10.5, $p=.008 ; \min F(1,43)=7.4, p=.01) \quad$ - the response times were longer with photographs $(561 \pm 13.1)$ compared to line-drawings $(548 \pm 13)$. The two-way interaction between the factors colour knowledge similarity and surface colour was marginally significant $(F 1(2,58)=4.5, p=.01 ; F 2(2$, $22)=4.6, p=.08 ; \min F(2,64)=2.3, p=.1$; Figure 3$)$. A post-hoc comparison (Tukey HSD) for the subject analysis showed that, when surface colour information was congruent there was a colour knowledge interference effect (similar colour knowledge $=575 \pm 15$; dissimilar colour knowledge $=559 \pm 13$; $p=.004)$. However, we did not observe a colour knowledge interference effect when the surface colour information was incongruent (similar colour knowledge $=544 \pm 13$; dissimilar colour knowledge $=543 \pm 13 ; p=.8$ ) or absent (similar colour knowledge $=556 \pm 14$; dissimilar colour knowledge $=551$ $\pm 12 ; p=.3$ )

\subsection{Discussion}

Together, the results indicate that the interaction between colour knowledge and surface colour information is strongly dependent on shape information. We only observed a colour knowledge interference effect in situations where the shape information activated by the distractor and by the target was similar. This corroborates the idea that colour and shape knowledge are stored in different systems within the object's structural description and for that reason can be independently activated. This result is in line with the neuropsychological evidence showing double dissociations between the shape and the colour knowledge systems (Luzzatti \& Davidoff, 1994; Miceli et al., 2001; Riddoch \& Humphreys, 1987). Moreover, the fact that we observed a strong colour knowledge interference effect, when shape is not enough to distinguish between two stimuli, gives support to the prediction that colour information is an important cue to reduce the competition at the level of the structural representation (Laws \& Hunter, 2006; Price \& Humphreys, 1989). The present findings show that the cognitive system relies on colour knowledge to resolve the competition and the effect of bottomup surface colour appears limited under these conditions. Participants took the same time to respond that a RED or a BROWN APPLE did not match the word TOMATO. In fact, when the shape information between the two stimuli was similar, there was little space surface colour information to intervene, corroborating the previous findings of Joseph and colleagues (Joseph, 1997; Joseph \& Proffitt, 1996). In these circumstances, the effects of surface colour were limited to situations where the colour knowledge between the stimuli was dissimilar and when the objects were presented as photographs. These results seems to indicate that, when shape information is not diagnostic of a certain object identity, (1) the effects of surface colour take place later compared to the effects of colour knowledge during recognition; (2) line-drawings and photographs differentially activate the surface and the colour knowledge systems. Line-drawings appear more effective in preventing the further bottom-up effects of surface colour information. 
Table 3. Significant main effects and interactions found in Experiment 2 (picture-word verification).

\begin{tabular}{lccc}
\hline & $\begin{array}{c}\text { Subject } \\
(\mathrm{F} 1)\end{array}$ & $\begin{array}{c}\text { Stimulus } \\
(\mathrm{F} 2)\end{array}$ & $\begin{array}{c}\text { Minimum F } \\
(\mathrm{min} F)\end{array}$ \\
\hline Shape & $<0.001$ & 0.001 & 0.001 \\
Colour knowledge & $<0.001$ & 0.01 & 0.01 \\
Surface colour & $<0.001$ & 0.03 & 0.07 \\
Shape $\times$ colour knowledge & $<0.001$ & 0.004 & 0.005 \\
Shape $\times$ colour knowledge & 0.02 & 0.003 & 0.07 \\
$\quad$ surface colour $\times$ stimulus & & & \\
$\quad$ type & & & \\
Follow-up comparisons & & & 0.005 \\
Similar shape condition & & 0.004 & 0.08 \\
$\quad$ Colour knowledge & $<0.001$ & 0.005 & \\
$\quad$ Colour knowledge $\times$ & 0.02 & & \\
$\quad$ surface colour $\times$ stimulus & & & 0.006 \\
type & & 0.002 & \\
Dissimilar shape condition & & 0.02 & \\
$\quad$ Surface colour & & & \\
$\quad$ Colour knowledge $\times$ & 0.001 & & \\
$\quad$ surface colour $\times$ stimulus & 0.01 & & \\
type & & &
\end{tabular}

The pattern of the results was somewhat different in situations where shape information between the distractor and the target was dissimilar. In these situations we did not observe a colour knowledge interference effect, but a surface colour interference effect, suggesting that the bottom-up effects of surface colour have a more prominent role during the recognition of diagnostic shape objects. It appears that the prototypical colour of the objects is not automatically activated. Instead it seems that the colour knowledge activation is dependent of the presence of the congruent surface colour. Participants took longer to say that a CHILI PEPPER did not match the word TOMATO only when the CHILI PEPPER was coloured in RED. When the CHILI PEPPER was coloured in an incongruent surface colour (BROWN) or when it was presented in an absent surface colour no colour knowledge interference effect was observed. In sum, our data suggest that colour knowledge information is not automatically activated, when shape information is diagnostic, and its activation is dependent upon the congruent surface colour displayed in the image. This result is consistent with our previous findings (Bramão et al., 2010).

\section{Experiment 2 (picture-word verification task)}

\subsection{Material and methods}

\subsubsection{Subjects}

Thirty new right-handed subjects took part in the experiment (mean age $[ \pm S D]=22.5 \pm 3.8$ years, range 18-35 years; mean years of education [ \pm SD] $=15.0 \pm 1.5$ years, range 13-20 years; 9 males and 21 females). All subjects were Portuguese native speakers with normal or corrected-to-normal vision; none report a history of colour vision problems, head injury or other neurological or psychiatric problems.

\subsubsection{Stimuli}

The stimuli used in this experiment are the same as used in Experiment 1.

\subsubsection{Procedure}

The experimental procedures were similar to the procedures of Experiment 1 with the exception that the object picture was presented first followed by the object name. Each trial begun with a fixation cross presented at the centre of the screen for 1250 ms. After the fixation cross, the object picture $(633 \times$ 498 pixels) was presented for $50 \mathrm{~ms}$, followed by a visual mask presented for $10 \mathrm{~ms}$. The object name (font Arial, font size 70) was presented on the screen immediately after the presentation of the visual mask and remained in the screen until participant's response (Figure 2). After 1500 ms a new trial started. Participants were instructed to decide as accurately and as quickly as possibly whether the picture and the name matched. The all procedure took about 90 minutes.

\subsubsection{Data analysis}

The data were analysed in the same manner as in Experiment 1.

\subsection{Results}

In total, $8.3 \%$ of the trials were excluded from the analysis $(1.2 \%$ two or more responses; $5.7 \%$ incorrect; $1.3 \%$ long; $0.06 \%$ short). In Table 3 we report the significant main effects and interactions found in the analysis. The response time analysis showed a significant shape similarity effect $(F 1(1,29)=$ 212.4, $p<.001 ; F 2(1,11)=21.5, p=.001 ; \min F(1$, $13)=19.5, p=.001) \quad-$ when shape information between the picture and the following word was similar the response times were longer $[718 \pm 15$ (Mean $\mathrm{RT} \pm \mathrm{SD}$ )] compared to the dissimilar case $(661 \pm 13)$. We also observed a significant effect of colour knowledge similarity $(F 1(1,29)=141.2, p$ $<.001 ; F 2(1,11)=9.3, p=.01 ; \min F(1,12)=8.7, p$ $=.01)-$ the response times were longer when the colour knowledge was similar (712 \pm 13.6$)$ compared 
to the dissimilar case $(667 \pm 15)$. The main effect of surface colour was also marginally significant $(F 1(2$, $58)=10.0, p<.001 ; F 2(2,22)=3.9, p=.03 ; \min F(2$, $40)=2.8, p=.07$ ) - a post-hoc comparison (Tukey HSD) for the subject analysis showed shorter response times when the surface colour was incongruent $(682 \pm 14)$ compared to congruent (696 \pm $14 ; p<.001)$ or absent $(691 \pm 14 ; p=.03)$. The twoway interaction between shape and colour knowledge was significant $(F 1(1,29)=124.6, p<.001 ; F 2$ $(1,11)=12.9, p=.004 ; \min F(1,13)=11.6, p=.005)$. A post-hoc comparison (Tukey HSD) for the subject analysis showed that when shape information between the name word and the picture was dissimilar there was no colour knowledge interference (similar colour knowledge $=663 \pm 13$; dissimilar colour knowledge $=660 \pm 14.2 ; p=.92$ ). On the other hand, when shape information between the two stimuli was similar there was a colour knowledge interference effect (similar colour knowledge $=761 \pm 15$; dissimilar colour knowledge $=674 \pm 15$; $p<.001)$. Additionally, the four-way interaction between all the factors was marginally significant $(F 1(2,58)=4.3, p=.02 ; \quad F 2(2,22)=7.7, p=.003$; $\min F(2,77)=2.8, p=.07)$. To further understand this interaction, we performed two separate analyses for each shape similarity dimension. The results from these two analyses are reported bellow.

\subsubsection{Similar shape}

The results showed the typical colour knowledge similarity effect $(F 1(1,29)=219.2, p<.001 ; F 2(1$, $11)=12.7, p=.004 ; \min F(1,12)=12.1, p=.005)-$ there were longer response times when colour knowledge was similar $(761 \pm 14.7)$ compared to the dissimilar case $(674 \pm 15)$. Additionally, the three-way interaction between colour knowledge, surface colour information and stimulus type was marginally significant $(F 1(2,58)=4.1, p=.02 ; F 2(2$, $22)=6.7, p=.005 ; \min F(2,76)=2.5, p=.08$; Figure 6). To further understand this three-way interaction, two separate analyses were performed for each stimulus type. Findings from Experiment 1 were replicated, the interaction between colour knowledge and surface colour information was only found for the photos $(F 1(2,58)=14.3, p<.001 ; F 2$ $(2,22)=11.4, p=.001 ; \min F(2,57)=6.3, p<.001) . \mathrm{A}$ post-hoc test (Tukey HSD) for the subject analysis reveal that when the colour knowledge activated by the picture and by the word object was similar there was no surface colour interference (congruent surface colour information $=754 \pm 16$; incongruent surface colour information $=766 \pm 16 ;$ absent surface colour information $=763 \pm 14 ; \quad p>.2$ ). However, when the colour knowledge activated by the picture and by the word was dissimilar, there was surface colour interference - subjects were quicker responding that the picture do not match with the following word when the surface colour was incongruent $(662 \pm 14.1)$ compared to congruent $(682 \pm 16.0 ; p<.001)$ or absent $(678 \pm 17.3 ; p$ $=.03)$.

\subsubsection{Dissimilar shape}

This analysis showed a surface colour effect $(F 1(2,58)$ $=16.4, p<.001 ; F 2(2,22)=8.7, p=.002 ; \min F(2,46)=$ $5.7, p=.006$ ) - a post-hoc comparison (Tukey HSD) for the subject analysis showed that the response times were longer when the surface colour was congruent compared to both incongruent or absent surface colour presentations (congruent surface colour = $675 \pm 14$; incongruent surface colour $=649 \pm 14$; absent surface colour $=661 \pm 13 ; p<.008$ ). The analysis also showed a marginal significant three-way interaction between the factors colour knowledge, surface colour information and stimulus type $(F 1(2$, 58) $=4.6$,

$p=.01 ; F 2(2,22)=5.4, p=.02 ; \quad \min F(2,68)=2.3$, $p=.09$; Figure 7). To further understand this threeway interaction, two separate analyses were performed for each stimulus type. These analyses showed a significant interaction between colour knowledge and surface colour only for the line-drawings $(F 1(2,58)=4.8, p=.009 ; F 2(2,22)=4.9, p=.01$; $\min F(2,64)=2.4, p=.1$ ). A post-hoc test (Tukey HSD) for the subject analysis showed a colour knowledge interference only when the stimuli were presented in a congruent surface colour (similar colour knowledge $=703 \pm 16$; dissimilar colour knowledge $=664 \pm 16 ; p=.03$ ). The results did not reveal a colour knowledge interference when the stimuli were presented in an incongruent (similar colour knowledge $=642 \pm 15.5$; dissimilar colour knowledge $=646 \pm 14 ; p=.6)$ or absent surface colour information (similar colour knowledge $=668 \pm 13.1$; dissimilar colour knowledge $=662 \pm 16 ; p=.2$ ).

\subsection{Discussion}

In general, the pattern of results replicates the results of Experiment 1. When shape information was insufficient to distinguish between the two stimuli, colour knowledge was automatically retrieved in order to resolve the competition from 


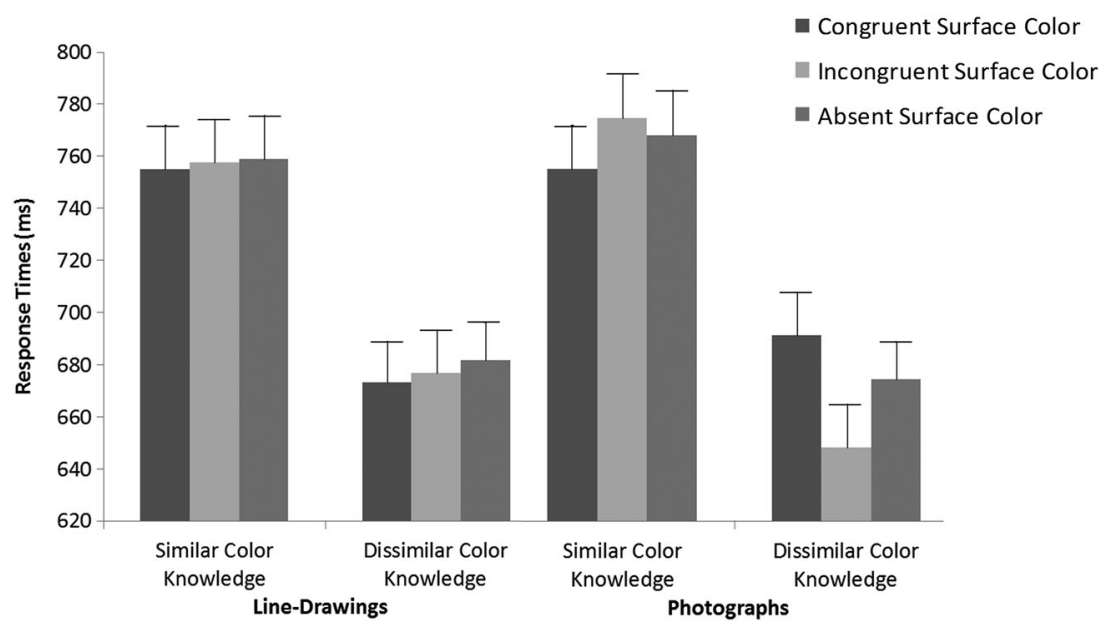

Figure 6. Three-way interaction between colour knowledge, surface colour and stimulus type $(F 1(2,58)=4.1, p=.02 ; F 2(2$, $68)=3.8, p=.03 ; \min F(2,126)=1.9, p=.1)$ found in Experiment 2 (PW verification task) when the shape information between the two stimuli was similar. Bars represent one standard error.

the non-diagnostic shapes. In contrast, when shape was sufficient to differentiate between the two stimuli, colour knowledge retrieval was triggered by surface colour in a bottom-up fashion. However, the congruent surface colour only triggered colour knowledge retrieval if the objects were presented as line-drawings. This result suggests that photographs and line-drawings differentially activate the surface colour and the colour knowledge systems.

\section{General discussion}

The main aim of the present study was to investigate the interaction between surface colour, colour knowledge, and shape information during object

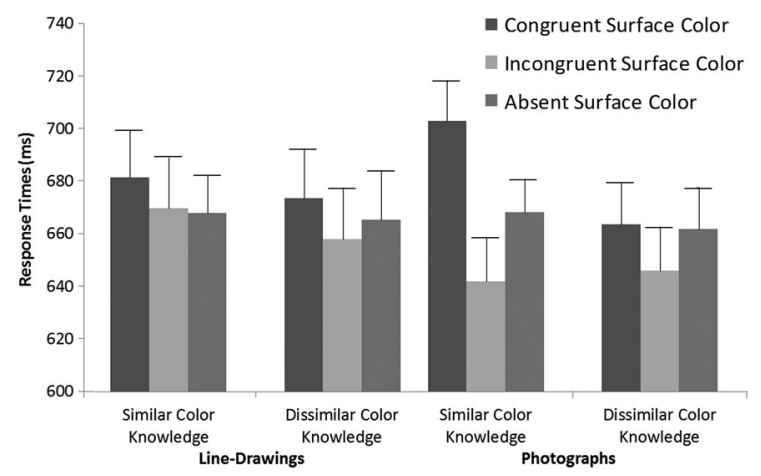

Figure 7. Three-way interaction between colour knowledge, surface colour and stimulus type $(F 1(2,58)=3.7, p$ $=.04 ; F 2(2,44)=3.2, p=.09 ; \min F(2,98)=1.7, p=.1)$ found in Experiment 2 ( $P W$ verification task) when the shape information between the pairs was dissimilar. Bars represent one standard error. recognition. In the non-matching trials, we manipulated the shape and colour information activated by the distractor and the target. We predicted that the greater similarity in terms of shape and colour between the two stimuli, the longer the participants would take to decide whether the two stimuli designated the same or a different identity. In both experiments, our results showed that the interaction between surface colour and colour knowledge is strongly dependent of shape information. In general, when shape information between the distractor and the target were similar we observed a more prominent role of colour knowledge. In contrast, when shape information related to the distractor and the target was dissimilar we observed a more prominent role of surface colour.

Because object recognition is a shape-driven system (Biederman, 1987; Marr \& Nishihara, 1978; see also, Scorolli \& Borghi, 2015) is not surprising that the interaction between the top-down colour knowledge and the bottom-up surface colour is mediated by shape information. Previous object recognition models have made a distinction between surface colour information and colour knowledge (Tanaka et al., 2001). The present findings extend previous results by showing that shape information is mediating whether colour knowledge or surface colour is more relevant to recognise a specific object. Previous research has also shown that there are other factors besides shape that mediate colour knowledge retrieval. For example, when context determines that colour knowledge is not relevant, colour knowledge retrieval might not be 
activated (Yee, Ahmed, \& Thompson-Schill, 2012). In Figure 8 we propose a processing account that shows the interaction between the surface and the colour knowledge system found in our study. The information presented in the figure does not imply that the processing or object recognition occurs in the serial fashion as depicted by the flowcharts or that the decisions were consciously made by our participants. Moreover, we do not intend to put forth a new theoretical model or framework to explain how colour information affects object recognition. The figure just means to represent the contribution of the various sources of information during the recognition processes according to our results. The most important sources of information during the decision processes are shown at top of the figure. The flowcharts terminate with the expected correct response from the subject. The further down on the chart the response appears the more interference/competition and the longer the response times.

Another main result of this study is related to the interactions between shape and colour knowledge: colour knowledge interference was only observed when shape information between distractor and target was similar. This result has two main implications. First, it shows that it is possible to access

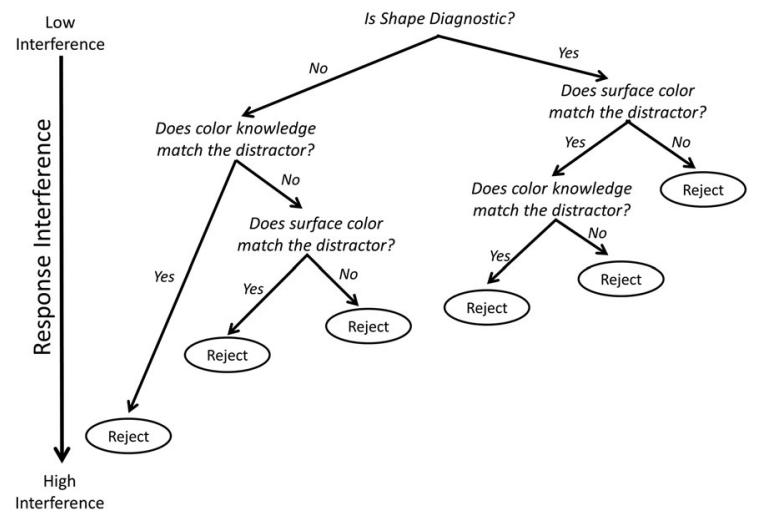

Figure 8. Processing account that fits the interactions between surface colour and colour knowledge found in our verification tasks. The most important sources of information during the decision processes and are shown on top of the figure. The flowcharts terminate with the expected correct response from the subject. The further down in the chart the response (REJECT) appears, the more interference/competition and the longer the response times. The role of surface colour during the recognition of a non-diagnostic shape is limited to situations were the objects were presented as photographs. Moreover, in Experiment 2, we observed that the colour knowledge during the recognition of diagnostic shaped objects was limited to situations were the objects were presented as line-drawings. object shape without accessing to object colour knowledge, indicating that shape and colour have distinct representations within the object's structural description forms. Some authors have suggested that the available evidence indicate that the structural representations corresponding to colour diagnostic objects are most likely stored in the longterm visual memory in a typical colour format (Bramão et al., 2012; Tanaka et al., 2001). The results reported here do not support this claim and are in line with the neuropsychological dissociations between shape and colour knowledge systems (Luzzatti \& Davidoff, 1994; Miceli et al., 2001; Riddoch \& Humphreys, 1987). Second, it also shows that the cognitive system relies on colour knowledge, and not on surface colour, to resolve the competition at the level of structural description, between objects that are similar in shape. Previous studies have shown that colour plays an important role in the recognition of objects that do not have a diagnostic or typical shape (Laws \& Hunter, 2006; Price \& Humphreys, 1989; Wurm et al., 1993). This study shows that it is colour knowledge, and not surface colour, that is automatically recruited in order to resolve the ambiguities imposed by non-diagnostic shapes. These effects are robust and were found in two different experimental verification tasks. In the first experiment we used a word-picture (WP) verification task and in the second experiment we used a picture-word (PW) verification task. Previous studies have shown differences in retrieving colour knowledge from words and pictures and the current idea in the literature is that colour knowledge retrieval is more effective with object names than with object pictures (Huettig \& Altmann, 2011; Naor-Raz et al., 2003). However, other studies have found that both colour patches and colour names can prime semantically related words in a similar way (Nijboer et al., 2006). Our results did not show any significant difference between the two verification tasks: the results of Experiment 2 basically replicated the results of Experiment 1, suggesting that the processes mediating the interaction between the shape and colour systems are very similar and not dependent of a specific object recognition task (for a similar result Joseph, 1997).

The findings here reported are also relevant for developmental psychology and the shape bias phenomenon. Developmental research have emphasised the importance of shape in children's early conceptualizations of the world (e.g. Landau, Smith, \& Jones, 1988). These studies show that, 
when learning labels for novel objects, children tend to extend object names on the basis of shape similarity rather than on colour or texture information (e.g. Graham, Williams, \& Huber, 1999). The present findings suggest that whatever attempt to explain the shape bias phenomena needs to take into consideration how shape information mediates colour knowledge retrieval.

\subsection{Recognising non-diagnostic shape objects}

Our results showed that during the recognition of non-diagnostic shaped objects, the effect of colour knowledge is more prominent than the effect of surface colour. In fact, we did not observe any evidence suggesting that surface colour can resolve the competition induced by shape- and colour knowledge similarity. This outcome suggests that colour knowledge retrieval is reinforced by the shape information creating high levels of interference that are solved through the recruitment of cognitive processes that do not make use of surface colour. In contrast, when the distractor and target shared shape, but not colour knowledge information, we found a surface colour interference effect for the photographs. Thus, in these circumstances, the interference resolution might benefit from surface colour information. When the visual system is confronted with a non-diagnostic shape several potential object representations are co-activated, as well as the prototypical colour of these representations, most likely by shape information itself. Our results suggest that, after shape, the cognitive system relies on colour knowledge to select the final representation. A grainer visual analysis of the input eliminates some object candidates, and if the colour knowledge does not impose an extra conflict, then surface colour is recruited in the decision process.

Moreover, and because the interference effect of surface colour was only observed with photographs and not with line-drawings, our data suggest that the surface colour and colour knowledge systems are differentially activated by these stimuli types. A previous study showed that the benefit of naming coloured photographs arise at an early visual recognition level, while the benefit of naming coloured line-drawings arise at the level of semantic representation (Zannino et al., 2010). It might thus be the case that line-drawings and photographs activate two different kinds of colour knowledge. Colour knowledge dissociations have been reported across the verbal and visual modalities, arguing for some kind of double representation of colour knowledge. For example, the patient RV could perform verbal tasks that involved retrieving the colour of abstract (e.g. "What colour name would you give communists?") and concrete (e.g. "What colour is a gherkin?") concepts, but he has unable to point to the correctly coloured picture of an object, unless he was allowed to rely on verbal mediation (Beauvois \& Saillant, 1985). Thus, his verbally associated knowledge of object colour seemed to be intact whereas his visual knowledge of colour was compromised. The opposite dissociation characterised the performance of KR (Hart \& Gordon, 1992). This patient could correctly discriminate correctly vs. incorrectly coloured animal pictures but could not verbally retrieve the correct colour of animals (e.g. "what colour is an elephant?"). We propose that line-drawings are more likely to activate a verballike colour knowledge system, while the photographs activate a visual-like colour knowledge system. The visual properties of the line-drawings make these stimuli more prone to activate the semantic representation of a certain object category (a token) and for that reason they can quickly activate semantic information about the object's identity, including its prototypical colour, independent of the perceptual input. On the other hand, photographs with natural colours, which might be harder to verbalise, are thus more likely to activate a particular object (a type). The presence of naturalistic colours in photographs might act as a visual cue to activate visual semantic information about the object, including, visual colour knowledge. So, when subjects are asked to verify whether a photograph of a COCONUT matches the word TOMATO, the visual properties of the objects are easily accessed and participants can also rely on the surface colour to resolve any conflict. This implies that the interaction between surface colour and colour knowledge occurs in the visual colour knowledge system. Consistent with this, it has been shown that colour knowledge can influence the colour perception of diagnostic colour objects (Witzel, Valkova, Hansen, \& Gegenfurtner, 2011).

\subsection{Recognising diagnostic shape objects}

Our results showed that during the recognition of a diagnostic shape object the bottom-up effects of surface colour are more prominent than the topdown effects of colour knowledge. Under these 
circumstances, the colour knowledge plays a limited role in recognition. When the shape between the distractor and target were dissimilar, then colour knowledge was not automatically activated. Subjects took longer to say that a picture of a CHILI PEPPER did not match the word TOMATO, only if the picture of the CHILI PEPPER was coloured in red. This result suggests that, when shape is diagnostic, the activation of colour knowledge is dependent on the congruency of the surface colour, and thus it seems that it is the surface colour information that prompts the retrieval of the prototypical object colour. For example, the surface colour RED, presented in the picture of the CHILI PEPPER, prompted the retrieval of its typical colour RED causing interference with the colour knowledge RED activated by the word TOMATO. An alternative explanation is that the surface colour RED of the CHILI PEPPER interfere with the colour knowledge RED activated by the name TOMATO. Even though these two alternatives are not mutually exclusive our results support the first explanation. If colour knowledge is not activated, then we should have found a surface colour interference effect when the colour knowledge between the pairs was dissimilar - for example, participants would have taken longer to say that a red image of a coconut did not match the word tomato compared to a brown image of a coconut. This was not the case. Thus, it seems that when the visual system is confronted with a diagnostic object shape, few or no competing object representations are activated, and for that, the recognition can continue without the need for any colour information. However, the perceptual colour depicted in the object image can, in a bottom-up fashion, prompt colour knowledge retrieval.

In Experiment 2, we observed that surface colour triggers colour knowledge retrieval only if the stimuli are presented as line-drawings. This result is consistent with our previous statement that linedrawings and photographs differentially activate the surface and the colour knowledge systems. We previously argued that colour knowledge activated by line-drawings is most likely in a verbal format. The surface colour of the line-drawings activated the verbal colour knowledge that further interferes with the colour knowledge activated by the target word (that is also more verbal-like). On the other hand, the non-naturalist colours of photograph stimuli offered a preferential cue to the visual colour knowledge system. Because the picture was just presented for a few milliseconds and immediately followed by the word target, no further interference with the verbal colour knowledge activated by the target word was observed. In Experiment 1, however, the picture was the last stimulus to be presented in a trial and the communication between the visual and the verbal colour knowledge systems could be established in a more efficient and complete way. Once more, this result seems to indicate that the surface colour and colour knowledge preferentially interact in the visual colour knowledge system. The verbal colour knowledge system is more easily accessed with line-drawings and is more resistant to the bottom-up influence of the surface colour.

\subsection{Conclusions}

This study clarifies the interaction between the colour knowledge and the surface colour systems and reconciles the somewhat contradictory results reported on the literature (Bramão et al., 2010; Huettig \& Altmann, 2011; Joseph, 1997; Joseph \& Proffitt, 1996). The main finding is that the interaction between these systems strongly depends on shape information. During the recognition of non-diagnostic shape objects, the top-down effects of colour knowledge are more prominent. In this situation, the colour knowledge is automatically activated in order to resolve the increased competition at the level of structural representation (description). In contrast, during the recognition of diagnostic shape objects the surface colour has a prominent role. In this case, surface colour information triggers, in a bottom-up fashion, colour knowledge retrieval. This study also shows that line-drawings and photographs differentially activate the surface and the colour knowledge systems. We suggest that line-drawings are more effective at triggering verbal colour knowledge, while photographs are most effective at triggering visual colour knowledge. Moreover, the interaction between surface colour and colour knowledge occur preferentially at the visual colour knowledge system.

\section{Disclosure statement}

No potential conflict of interest was reported by the authors.

\section{Funding}

This work was supported by Max Planck Institute for Psycholinguistics, Donders Institute for Brain, Cognition and 
Behaviour, Fundação para a Ciência e Tecnologia (FCT; UID/BIM/04773/2013 CBMR).

\section{References}

Beauvois, M. F., \& Saillant, B. (1985). Optic aphasia for colours and colour agnosia: A distinction between visual and visuo-verbal impairments in the processing of colours. Cognitive Neuropsychology, 2, 1-48. doi:10. 1080/02643298508252860

Biederman, I. (1987). Recognition-by-components: A theory of human image understanding. Psychological Review, 94, 115-117. doi:10.1037/0033-295X.94.2.115

Bramão, I., Faísca, L., Forkstam, C., Inácio, F., Araújo, S., Petersson, K. M., \& Reis, A. (2012). The interaction between surface color and color knowledge: Behavioural and electrophysiological evidence. Brain and Cognition, 78, 28-37.

Bramão, I., Faísca, L., Petersson, K. M., \& Reis, A. (2010). The influence of surface color information and color knowledge information in object recognition. American Journal of Psychology, 123, 459-468.

Brodie, E., Wallace, A., \& Sharrat, B. (1991). Effect of surface characteristics and style of production on naming and verification of pictorial stimuli. The American Journal of Psychology, 104, 517-545. doi:10.2307/1422939

Clark, H. (1973). The language-as-fixed-effect fallacy: A critique of language statistics in psychological research. Journal of Verbal Learning and Verbal Behavior, 12, 335-359. doi:10.1016/S0022-5371(73)80014-3

Gegenfurtner, K. (2003). Cortical mechanisms of colour vision. Nature Reviews Neuroscience, 4, 563-572. doi:10. 1038/nrn1138

Graham, S. A., Williams, L. D., \& Huber, J. F. (1999). Preschoolers' and adults' reliance on object shape and object function for lexical extension. Journal of Experimental Child Psychology, 74, 128-151. doi:10. 1006/jecp.1999.2514

Hart, J., \& Gordon, B. (1992). Neural subsystems for object knowledge. Nature, 359, 60-64. doi:10.1038/359060a0

Hodges, J. R., Salmon, D. P., \& Butters, N. (1992). Semantic memory impairment in Alzheimer's disease: Failure of access or degraded knowledge? Neuropsychologia, 30, 301-314. doi:10.1016/0028-3932(92)90104-T

Huettig, F., \& Altmann, G. T. M. (2011). Looking at anything that is green when hearing "frog": How object surface colour and stored object colour knowledge influence language-mediated overt attention. The Quarterly Journal of Experimental Psychology, 64, 122-145. doi:10.1080/17470218.2010.481474

Humphreys, G. W., Lloyd-Jones, T. J., \& Fias, W. (1995). Semantic interference effects on naming using a postcue procedure: Tapping the links between semantics and phonology with pictures and words. Journal of Experimental Psychology: Learning, Memory and Cognition, 21, 961-980. doi:10.1037/0278-7393.21.4.961

Humphreys, G. W., Riddoch, M. J., \& Quinlan, P. (1988). Cascade processing in picture identification. Cognitive Neuropsychology, 5, 67-104. doi:10.1080/0264329880 8252927
Joseph, J. (1997). Color processing in object verification. Acta Psychologica, 97, 95-127. doi:10.1016/S0001-6918 (97)00026-7

Joseph, J., \& Proffitt, D. (1996). Semantic versus perceptual influences of color in object recognition. Journal of Experimental Psychology: Learning, Memory and Cognition, 22, 407-429. doi:10.1037/0278-7393.22.2.407

Landau, B., Smith, L. B., \& Jones, S. S. (1988). The importance of shape in early lexical learning. Cognitive Development, 3, 299-321. doi:10.1016/0885-2014(88)90014-7

Laws, K., \& Hunter, M. Z. (2006). The impact of colour, spatial resolution, and presentation speed on category naming. Brain and Cognition, 62, 89-97. doi:10.1016/j. bandc.2006.03.002

Lloyd-Jones, T. J., \& Nakabayashi, K. (2014). Long-term repetition priming and semantic interference in a lexicalsemantic matching task: Tapping the links between object names and colors. Frontiers in Psychology, 5, 1-10.

Luzzatti, C., \& Davidoff, J. (1994). Impaired retrieval of object-color knowledge with preserved color naming. Neuropsychologia, 32, 933-950. doi:10.1016/0028-3932 (94)90044-2

Marr, D., \& Nishihara, H. (1978). Representation and recognition of the spatial organization of three-dimensional shapes. Proceedings of the Royal Society B: Biological Sciences, 200, 269-294. doi:10.1098/rspb.1978.0020

Miceli, G., Fouch, E., Capasso, R., Shelton, J. R., Tomaiuolo, F., \& Caramazza, A. (2001). The dissociation of color from form and function knowledge. Nature Neuroscience, 4, 662-667. doi:10.1038/88497

Naor-Raz, G., Tarr, M. J., \& Kersten, D. (2003). Is color an intrinsic property of object representation? Perception, 32, 667-680. doi:10.1068/p5050

Nijboer, T. C. W., van Zandvoort, M. J. E., \& de Haan, E. H. F. (2006). Seeing red primes tomato: Evidence for comparable priming from colour and colour name primes to semantically related word targets. Cognitive Processing, 7, 269-274. doi:10.1007/s10339-006-0153-8

Price, C., \& Humphreys, G. W. (1989). The effects of surface detail on object categorization and naming. The Quarterly Journal of Experimental Psychology Section A, 41, 797-828. doi:10.1080/14640748908402394

Raaijmakers, J. (2003). A further look at the "Language-asfixed-effect fallacy". Canadian Journal of Experimental Psychology, 57, 141-151. doi:10.1037/h0087421

Raaijmakers, J., Schrijnemakers, J., \& Gremmen, F. (1999). How to deal with "The language-as-fixed-effect fallacy": Common misconceptions and alternative solutions. Journal of Memory and Language, 41, 416-426. doi:10.1006/jmla.1999.2650

Reis, A., Faísca, L., Ingvar, M., \& Petersson, K. M. (2006). Color makes a difference: Two-dimensional object naming in literate and illiterate subjects. Brain and Cognition, 60, 49-54. doi:10.1016/j.bandc.2005.09.012

Riddoch, M. J., \& Humphreys, G. W. (1987). Visual object processing in optic aphasia: A case of semantic access agnosia. Cognitive Neuropsychology, 4, 131-185. doi:10. 1080/02643298708252038

Scorolli, C., \& Borghi, A. M. (2015). Square bananas, blue horses: The relative weight of shape and color in concept recognition and representation. [Original 
Research]. Frontiers in Psychology, 6. doi:10.3389/fpsyg. 2015.01542

Tanaka, J., Weiskopf, D., \& Williams, P. (2001). The role of color in high-level vision. Trends in Cognitive Sciences, 5, 211-215. doi:10.1016/S1364-6613(00)01626-0

Uttl, B., Graf, P., \& Santacruz, P. (2006). Object color affects identification and repetition priming. Scandinavian Journal of Psychology, 47, 313-325. doi:10.1111/j.14679450.2006.00532.x

Vitkovitch, M., Cooper-Pye, E., \& Leadbetter, A. G. (2006). Semantic priming over unrelated trials: Evidence for different effects in word and picture naming. Memory and Cognition, 34, 715-725. doi:10.3758/BF03193590

Witzel, C., Valkova, H., Hansen, T., \& Gegenfurtner, K. R. (2011). Object knowledge modulates colour appearance. Iperception, 2, 13-50.
Wurm, L. H., Legge, G. E., Isenberg, L. M., \& Luebker, A. (1993). Color improves object recognition in normal and low vision. Journal of Experimental Psychology: Human Perception and Performance, 19, 899-911. doi:10.1037/0096-1523.19.4.899

Yee, E., Ahmed, S. Z., \& Thompson-Schill, S. L. (2012). Colorless green ideas (can) prime furiously. Psychological Science, 23, 364-369. doi:10.1177/ 0956797611430691

Zannino, G. D., Perri, R., Salamone, G., Lorenzoa, C. D., Caltagirone, C., \& Carlesimo, G. (2010). Manipulating color and other visual information influences picture naming at different levels of processing: Evidence from Alzheimer subjects and normal controls. Neuropsychologia, 48, 2571-2578. doi:10.1016/j. neuropsychologia.2010.05.003 